\title{
Safety and Efficacy of Procarbazine and Lomustine Chemotherapy as a Salvage Treatment for Recurrent Adult Glioma
}

\section{Stephen Ahn}

The Catholic University of Korea

\section{Young II Kim}

The Catholic University of Korea

Ja Young Shin

The Catholic University of Korea

Jae-Sung Park

The Catholic University of Korea

Changyoung Yoo

The Catholic University of Korea

\section{Youn Soo Lee}

The Catholic University of Korea

\section{Yong-Kil Hong}

The Catholic University of Korea

\section{Sin-Soo Jeun}

The Catholic University of Korea

Seung-Ho Yang ( $\sim$ 72ysh@catholic.ac.kr)

St. Vincent's Hospital, The Catholic University of Korea https://orcid.org/0000-0002-3490-1064

\section{Research Article}

Keywords: Recurrent glioma, PCV chemotherapy, procarbazine, lomustine, toxicity

Posted Date: September 9th, 2021

DOl: https://doi.org/10.21203/rs.3.rs-878192/v1

License: (c) (i) This work is licensed under a Creative Commons Attribution 4.0 International License.

Read Full License 


\section{Abstract}

\section{Purpose}

While procarbazine, lomustine, and vincristine (PCV) chemotherapy is considered a salvage option for adult glioma, whether vincristine included in this regimen is beneficial is uncertain due to its potential toxicity and uncertain efficacy. In this study, we evaluated the safety and efficacy of PC chemotherapy in contrast with those of PCV chemotherapy.

\section{Methods}

Using electronic medical records, all patient with adult recurrent glioma who received PC or PCV chemotherapy between 2009 and 2020 at Seoul St. Mary's Hospital or St. Vincent's Hospital were examined retrospectively. A total of 59 patients met our eligibility criteria. Among them, 15 patients

received PC chemotherapy (PC group) and 44 patients received PCV chemotherapy (PCV group).

\section{Results}

The PC group presented a significantly lower hematology toxicity (anemia: $6.7 \%$ vs. $45.5 \% ; p=$ 0.02 and thrombocytopenia: $20.0 \%$ vs. $70.4 \% ; p<0.001$ ). Also, the clinical impacts of PC chemotherapy, including delay of a cycle, dose reduction, discontinuation of drug(s), or total cessation of chemotherapy, were significantly less frequent $(26.7 \%$ vs. $68.2 \% ; p=0.012)$. The overall survival of PC group was significantly longer than that of PCV group (396 vs. 232 days; $p=0.042$ ), while there was no significant difference in progression-free survival between two groups (284.5 vs. 131 days; $p=0.077$ ).

\section{Conclusion}

This is the first comparative study to suggest that PC chemotherapy leads to less toxicity than PCV chemotherapy without loss of clinical efficacy in patients with recurrent adult glioma. Further prospective and larger studies are needed to validate our findings.

\section{Introduction}

Glioma is the most common and most malignant brain tumor in adults, composing most of all brain malignancy diagnoses in this population[1, 2]. Typically, the clinical outcomes of this condition are devastating, although aggressive multimodal treatments, including surgery, radiotherapy, and chemotherapy-which mainly is composed of drugs in the temozolomide (TMZ) and nitrosourea classes -can have some effect $[3,4]$. For cases of recurrent glioblastoma, which is the most common and most malignant type of glioma, almost all patients eventually experience a recurrence and die within six months after diagnosis of recurrence[5, 6].

There is no consensus regarding salvageable options for recurrent and TMZ-resistant adult glioma[7-10]. Bevacizumab has shown significant clinical responses in some patients; however, identifying good 
responders and predicting the clinical outcome in the overall population remain difficult[11, 12]. Procarbazine, lomustine, and vincristine (PCV) chemotherapy has long been trialed as one of the salvageable options for recurrent adult gliomas[13-16]. However, the toxicity of vincristine, including peripheral neurotoxicity frequently seen in neuro-oncology clinics, has been documented, resulting in its reduction or discontinuation in many outpatients[17-19]. In addition to its toxicity, vincristine is composed of relatively heavy molecules (825 Daltons), and concerns exist about its successful crossing of the blood-brain barrier[20]. In this context, a few studies recently have trialed modified procarbazine and lomustine (PC) therapy protocols for recurrent gliomas[21-24]. However, to the best of our knowledge, no study has yet compared the safety and efficacy of PC chemotherapy and PCV chemotherapy in a single study cohort.

In this study, we evaluated whether PC chemotherapy is less toxic than PCV chemotherapy and whether the survival outcomes of PC chemotherapy are noninferior to those of PCV chemotherapy in patients with recurrent adult glioma, whose data were collected retrospectively from two hospitals.

\section{Methods}

\section{Study population}

This multicenter retrospective study was approved by the Institutional Review Board of Seoul St. Mary's Hospital and St. Vincent's Hospital. The electronic medical records of adult glioma patients treated at these two hospitals between 2010 and 2020 were examined. The study inclusion criteria were (1) glioma pathologically confirmed by craniotomy or biopsy, (2) glioma recurrence confirmed radiologically and/or pathologically, (3) receipt of PC or PCV chemotherapy following recurrence diagnosis, and (4) accessible baseline clinical variables and survival data. The study exclusion criteria were (1) received PC or PCV chemotherapy as adjuvant therapy after initial diagnosis and (2) a medical history of hematologic or rheumatologic disease. A flow of the study design is presented in Fig. 1.

\section{Treatment Protocol}

After maximal safe resection at initial surgery, we performed adjuvant therapy following the best treatment protocol(s) at each institution. If the diagnosis was glioblastoma, we performed concomitant chemoradiation (TMZ dose: $75 \mathrm{mg} / \mathrm{m}^{2}$ ) and six cycles of adjuvant TMZ chemotherapy (TMZ dose: 150$200 \mathrm{mg} / \mathrm{m}^{2}$ ). If the diagnosis was grade II or III glioma, we conducted adjuvant radiotherapy, in which the dosage was either 5,940 cGy for 33 fractions or 6,000 cGy for 30 fractions. In the case of grade III glioma, we added adjuvant chemotherapy of PCV or PC chemotherapy. When recurrence occurred, the first chemotherapy considered was often TMZ; when the use of TMZ chemotherapy was not possible due to various reasons, such as prior history of TMZ administration within 6 months, and swallowing difficulty, then bevacizumab or nitrosourea-based chemotherapy was considered. 
At St. Vincent's Hospital, patients with recurrent adult glioma received PC chemotherapy instead of PCV chemotherapy. PC chemotherapy was composed of lomustine $\left(75 \mathrm{mg} / \mathrm{m}^{2}\right.$, day 1$)$ and procarbazine $\left(60 \mathrm{mg} / \mathrm{m}^{2}\right.$, days $\left.11-24\right)$ administered orally every four weeks. This modified protocol was discussed in a previous study of one of our authors[21]. At Seoul St. Mary's Hospital, PCV chemotherapy was administered to recurrent glioma patients, with lomustine $\left(110 \mathrm{mg} / \mathrm{m}^{2}\right.$, day 1$)$ and procarbazine $(60$ $\mathrm{mg} / \mathrm{m}^{2}$, days $8-21$ ) administered orally but vincristine administered intravenously $\left[1.4 \mathrm{mg} / \mathrm{m}^{2}\right.$ (maximum of $2 \mathrm{mg}$ ), days 8 and 29] every six weeks.

\section{Clinical Variables}

The clinical variables of sex; age; pathological diagnosis, including molecular features; prior history of surgery, radiation, or chemotherapy; radiological findings; performance status; and survival status and/or death date were collected. Diagnosis of recurrent glioma was performed by two neuropathologists according to the 2016 World Health Organization classification of the central nervous system. IDH mutation was evaluated by immunohistochemistry or directing sequencing. If necessary, IDH 2 mutation was evaluated by directing sequencing. The presence of a 1p19q co-deletion was examined using fluorescence in situ hybridization. The $0^{6}$-methylguanine-DNA-methyltransferase (MGMT) gene methylation status was evaluated by polymerase chain reaction. Performance status was estimated according to the scale of the Eastern Cooperative Oncology Group (ECOG). All kinds of toxicities were evaluated according to the Common Terminology Criteria for Adverse Events (CTCAE) version 5.0. Also, the related impact of the toxicity of PC or PCV chemotherapy on the course of the treatment schedule was classified into four categories: delay of a cycle, dose reduction, discontinuation of drug(s), or total cessation of chemotherapy. Radiographic responses on magnetic resonance imaging (MRI) were determined by specialized neuroradiologists according to the response assessment in neuro-oncology (RANO) criteria. The date of recurrence was defined as the date of MRI showing recurrence. Survival status and/or death date were collected from the Korea Central Cancer Registry database.

\section{Statistical analysis}

The overall survival (OS) after recurrence was defined as days from the starting date of PC or PCV chemotherapy to death, while progression-free survival (PFS) was defined as days from the starting date of PC or PCV chemotherapy to disease progression was confirmed by MRI. Patients who were confirmed to be alive on March 31, 2021, were censored. The mean duration of follow-up was 424.6 days (range: 55-2,491 days). All clinical variables were considered with descriptive statistics. The differences of clinical variables between the two treatment groups were compared using Fisher's exact test or the Chisquare test. The normality test was performed for continuous variables. Kaplan-Meier survival analysis and the log-rank test were used to calculate the median OS and PFS values of the groups. All statistical analyses were conducted using the $\mathrm{R}$ version 4.0.5 software program ( $\mathrm{R}$ Foundation for Statistical Computing, Vienna, Austria). 


\section{Results}

\section{Patient characteristics}

Among a total of 59 patients enrolled in this study, 15 received PC chemotherapy (PC group) and 44 patients received PCV chemotherapy (PCV group) as salvage treatment for recurrent gliomas. Clinical characteristics, including sex, age, initial diagnosis, IDH mutation, 1p19q co-deletion, MGMT promoter methylation, and prior history of radiotherapy were not statistically different between the two groups. However, the median interval from radiation to chemotherapy in the PC group was shorter than that of the PCV group [4.0 (range: $0-42$ ) months vs. 22.0 (range: $0-167$ ) months, $p=0.004$ ]. Also, fewer patients in the PC group had prior history of any chemotherapy (33.3\% vs. $84.7 \% ; p=0.003)$, while there was no difference in the median interval from the last chemotherapy session to initiation of PC or PCV chemotherapy between the two groups. The summarized baseline characteristics of these patients are described in Table 1. 
Table 1

Baseline characteristics of patients with recurrent adult glioma who received PC or PCV chemotherapy

\begin{tabular}{|c|c|c|c|}
\hline & $\begin{array}{l}\text { PC group }(n \\
=15)\end{array}$ & $\begin{array}{l}\text { PCV group }(n \\
=44)\end{array}$ & $\begin{array}{l}p- \\
\text { value }\end{array}$ \\
\hline Male sex, n (\%) & $11(73.3)$ & $27(61.4)$ & 0.600 \\
\hline Age at chemotherapy, years, n (range) & $52.2(20-79)$ & $\begin{array}{l}49.6 \text { (range } \\
21-73)\end{array}$ & 0.528 \\
\hline Initial diagnosis, n (\%) & & & $\overrightarrow{0} .999$ \\
\hline GBM & $5(33.3)$ & $14(31.8)$ & \\
\hline Non-GBM & $10(66.7)$ & $30(68.2)$ & \\
\hline IDH mutation, n (\%) & & & 0.107 \\
\hline Yes & $3(20.0)$ & $7(15.9)$ & \\
\hline No & $10(66.7)$ & $18(40.9)$ & \\
\hline Unknown & $2(13.3)$ & $19(43.2)$ & \\
\hline 1p19q co-deletion, n (\%) & & & 0.392 \\
\hline Yes & $2(13.3)$ & $7(15.9)$ & \\
\hline No & $11(73.3)$ & $24(54.5)$ & \\
\hline Unknown & $2(13.3)$ & $13(29.5)$ & \\
\hline MGMT methylation, $\mathrm{n}(\%)$ & & & 0.311 \\
\hline Yes & $8(53.4)$ & $16(36.4)$ & \\
\hline No & $4(26.7)$ & $16(36.4)$ & \\
\hline Unknown & $3(20.0)$ & $12(27.3)$ & \\
\hline Prior radiation therapy, $\mathrm{n}(\%)$ & & & $\overrightarrow{0.999}$ \\
\hline Yes & $15(10.0)$ & $43(97.7)$ & \\
\hline No & $0(0.0)$ & $1(2.3)$ & \\
\hline $\begin{array}{l}\text { Median interval from radiation to PC or PCV, months, } \mathrm{n} \\
\text { (range) }\end{array}$ & $4.0(0-42)$ & $22.0(0-167)$ & 0.004 \\
\hline \multicolumn{3}{|l|}{ Prior chemotherapy, n (\%) } & $\begin{array}{l}< \\
0.003\end{array}$ \\
\hline $\begin{array}{l}\text { ECOG, Eastern Cooperative Oncology Group; GBM, gliob } \\
\text { methyltransferase; PC, procarbazine and lomustine; PC } \\
\text { TMZ, temozolomide }\end{array}$ & $\begin{array}{l}\text { oma; MGMT, O } \\
\text { carbazine, lon }\end{array}$ & $\begin{array}{l}\text { nethylguanine } \\
\text { stine, and vinc }\end{array}$ & \\
\hline
\end{tabular}




\begin{tabular}{|c|c|c|c|}
\hline & $\begin{array}{l}\text { PC group ( } \\
=15)\end{array}$ & $\begin{array}{l}\text { PCV group ( } \\
=44)\end{array}$ & $\begin{array}{l}p- \\
\text { value }\end{array}$ \\
\hline Never & $10(66.7)$ & $7(16.3)$ & \\
\hline TMZ & 5 (33.3) & $30(69.8)$ & \\
\hline TMZ, bevacizumab & $0(0.0)$ & $1(2.3)$ & \\
\hline TMZ, PCV & $0(0.0)$ & $5(11.6)$ & \\
\hline $\begin{array}{l}\text { Median interval from last chemotherapy to PC or PCV, } \\
\text { months, } n \text { (range) }\end{array}$ & $1.0(0-13)$ & $2.0(0-61)$ & 0.610 \\
\hline ECOG score, n (\%) & & & 0.311 \\
\hline $0-1$ & $7(46.7 \%)$ & $29(65.9 \%)$ & \\
\hline$\geq 2$ & $8(53.3 \%)$ & $15(34.1 \%)$ & \\
\hline
\end{tabular}

\section{Toxicity experiences and the impact of toxicity on chemotherapy schedule}

The PC group presented a significantly lower hematology toxicity profile (anemia: $6.7 \%$ vs. $45.5 \% ; p=$ 0.017 and thrombocytopenia: $20.0 \%$ vs. $70.4 \% ; p<0.001$ ) and liver function (elevated liver enzymes: $0 \%$ vs. $25.0 \% ; p=0.078$ ). CTCAE grade III or IV toxicities were less frequently observed in the PC group, although there was no statistically significant difference. Rates of other toxicities, including neutropenia, kidney injury, allergic skin reactions, and peripheral neurotoxicity, were not significantly different between the two groups. Detailed information about toxicities is presented in Table 2. 
Table 2

Toxicity in patients with recurrent adult glioma who received PC or PCV chemotherapy

\begin{tabular}{|c|c|c|c|c|c|c|}
\hline & \multicolumn{3}{|c|}{ All grades* } & \multicolumn{3}{|c|}{ Grades III and IV* } \\
\hline & $\begin{array}{l}\text { PC } \\
\text { group }\end{array}$ & $\begin{array}{l}\text { PCV } \\
\text { group }\end{array}$ & $\begin{array}{l}p- \\
\text { value }\end{array}$ & $\begin{array}{l}\text { PC } \\
\text { group }\end{array}$ & $\begin{array}{l}\text { PCV } \\
\text { group }\end{array}$ & $p$-value \\
\hline & $(n=15)$ & $(n=44)$ & & $(n=15)$ & $(n=44)$ & \\
\hline Anemia, n (\%) & $1(6.7)$ & $20(45.5)$ & 0.017 & $1(6.7)$ & $6(13.6)$ & 0.796 \\
\hline Neutropenia, n (\%) & $3(20.0)$ & $17(38.6)$ & 0.317 & $3(20.0)$ & $8(18.2)$ & $>0.999$ \\
\hline Thrombocytopenia, n (\%) & $3(20.0)$ & $31(70.4)$ & $<.001$ & $3(20.0)$ & $14(31.8)$ & 0.587 \\
\hline $\begin{array}{l}\text { Elevated liver enzymes, } \mathrm{n} \\
(\%)\end{array}$ & $0(0.0)$ & $11(25.0)$ & 0.078 & $0(0.0)$ & $6(13.6)$ & 0.310 \\
\hline Elevated creatinine, $\mathrm{n}(\%)$ & $0(0.0)$ & $2(4.6)$ & 0.989 & $0(0.0)$ & $1(2.3)$ & $>0.999$ \\
\hline Allergic reaction, n (\%) & $0(0.0)$ & $1(2.3)$ & $\begin{array}{l}> \\
0.999\end{array}$ & $0(0.0)$ & $0(0.0)$ & $>0.999$ \\
\hline $\begin{array}{l}\text { Peripheral } \\
\text { neurotoxicity, n (\%) }\end{array}$ & $0(0.0)$ & $5(11.4)$ & 0.408 & $0(0.0)$ & $0(0.0)$ & $>0.999$ \\
\hline
\end{tabular}

We describe the adverse impacts of toxicity on chemotherapy schedule in Table 3. The PC group significantly less frequently experienced any of delay of cycle, dose reduction, discontinuation of one of the chemotherapeutic drugs, or cessation of the entire chemotherapy regimen than did the PCV group ( $26.7 \%$ vs. $68.2 \% ; p=0.012$ ). Each type of toxicity was less frequently observed in the PC group, although this result failed to show statistical significance.

Table 3

Adverse impacts of the toxicity of PC or PCV on the course of chemotherapy for recurrent adult glioma

\begin{tabular}{|llll|}
\hline & PC group $(\mathrm{n}=15)$ & PCV group $(\mathrm{n}=44)$ & $p$-value \\
\hline Total, $\mathrm{n}(\%)$ & $4(26.7)$ & $30(68.2)$ & 0.012 \\
\hline Delay of a cycle, $\mathrm{n}(\%)$ & $3(20.0)$ & $14(31.8)$ & 0.587 \\
\hline Dose reduction, $\mathrm{n}(\%)$ & $1(6.7)$ & $6(13.6)$ & 0.796 \\
\hline Drug discontinuation, $\mathrm{n}(\%)$ & $0(0.0)$ & $8(18.2)$ & 0.180 \\
\hline Chemotherapy cessation, $\mathrm{n}(\%)$ & $0(0.0)$ & $2(4.5)$ & 0.989 \\
\hline
\end{tabular}

Comparison of clinical outcomes between the PC and PCV groups 
The OS of the PC group was significantly longer than that of the PCV group (396 vs. 232 days; $p=0.042$ ), while there was no significant difference in PFS between the two groups (284.5 vs. 131 days; $p=0.077$ ). The Kaplan-Meier survival curves of OS and PFS in the two groups are illustrated in Fig. 2. We also conducted a subgroup analysis according to initial diagnosis. In the nonglioblastoma subgroup, the OS of the PC group was significantly better than that of the PCV group (514 vs. 250 days; $p=0.037$ ), while there was no significant difference in PFS between the two groups (442 vs. 174 days; $p=0.163$ ). In the glioblastoma subgroup, there were no significant differences in OS and PFS between the two groups (OS: 154 vs. 190 days; $p=0.671$ and PFS: 102 vs. 87 days; $p=0.310$ ). The Kaplan-Meier survival curves of OS and PFS in the two subgroups are illustrated in Supplementary Figs. 1 and 2.

\section{Discussion}

In our retrospective and comparative study between two institutions, we tried to compare not only the safety, but also the efficacy of PC chemotherapy compared to those of PCV chemotherapy using electronic medical records from tertiary hospitals in Koreans. We also tried to evaluate how the toxicity of these chemotherapies affected the course of chemotherapy in patients with recurrent adult glioma. Our findings showed that anemia and thrombocytopenia were significantly more frequent in PCV groups than in the PC groups (anemia: $45.5 \%$ vs. $6.7 \% ; p=0.017$ and thrombocytopenia: $70.4 \%$ vs. $20.0 \% ; p<0.001$, respectively). Anemia of higher than CTCAE grade III was also more frequent in the PCV group than the PC group. Peripheral neurotoxicity, which is a major concern regarding vincristine, was more frequently observed in the PCV group than the PC group ( $0.0 \%$ vs. $11.4 \%)$, as expected, however, there was no statistical significance $(p=0.408)$. In addition, frequent and severe adverse events in the PCV group also resulted in greater disruption to the course of chemotherapy, such as delay of a cycle, dose reduction, discontinuation of vincristine, and cessation of salvage chemotherapy $(68.2 \%$ vs. $26.7 \% ; p=0.012)$. In contrast, regarding concerns about inferior efficacy when omitting vincristine, our findings suggest that survival outcomes were not different between the two groups. Interestingly, the OS of the PC group was significantly superior to that of the PCV group (396 vs. 232 days; $p=0.042$ ), while the PFS of the PC group was not different from that of the PCV group (284.5 vs. 131 days; $p=0.077$ ). This can be explained by numerous studies showing that the occurrence of less toxicity after chemotherapy correlates with better prognosis[25, 26].

When considering chemotherapeutic drugs for recurrent glioma, there have been options identified to date, including TMZ rechallenged or continuously administered with low-dose, bevacizumab, and PCVbased chemotherapy $[4,11,12,27,28]$. As a salvage therapy after TMZ for recurrent glioma, numerous clinical trials have assessed the efficacy of PCV-based chemotherapy $[13,14,16]$. However, in clinics, toxicities including hematologic, neurologic, liver, kidney, and skin problems were diagnosed frequently and sometimes very severe, which is a major hindrance when choosing PCV chemotherapy as salvage therapy for recurrent glioma patients, especially in those who are elderly or with a lower performance 
status[17, 29]. In addition, there have been concerns about the efficacy of vincristine because its molecular weight (825 Daltons) might be too high to penetrate the blood-brain barrier[20].

In this context, a few studies have put forth the idea of adopting a modified PC-based chemotherapy regimen without vincristine for glioma patients[21-24]. Vesper et al. retrospectively analyzed clinical outcomes and toxicities of 315 patients with oligodendroglial brain tumors who received PCV or PC chemotherapy as adjuvant treatment after surgical resection and radiation. Their study showed that the PFS of patients who received PC chemotherapy was not different from that of patients who received PCV chemotherapy, with significantly fewer hematologic and neurological toxicities[23]. Webre et al. also evaluated 97 patients with primary anaplastic oligodendroglioma who received PCV or PC chemotherapy as adjuvant treatment, reporting that the clinical outcomes of PC chemotherapy for primary anaplastic oligodendroglial tumors were not different from those of patients who received PCV chemotherapy, with lower hematologic toxicities[22].

To the best of our knowledge, this is the first study to compare the safety and efficacy of PC and PCV chemotherapy as salvage treatment in recurrent glioma patients. In accordance with two previous studies exploring the use of PC chemotherapy in primary oligodendroglial patients[22, 23], we have added some evidence that PC chemotherapy is as beneficial as PCV chemotherapy but with significantly less toxicity due to omitting vincristine. Taken together, we suggest that PC chemotherapy can be an alternative option to PCV chemotherapy, especially for use in patients expected to be intolerable to PCV chemotherapy, including elderly patients or those with lower performance.

Our study should be considered within the scope of several limitations. First, contrary to previous studies designed to compare the efficacy and safety of PC chemotherapy with those of PCV chemotherapy, our study included heterogeneous recurrent gliomas, which can better reflect real-world situations but cause bias due to the heterogeneity of the study population. Second, although several baseline characteristics, including initial diagnosis, molecular features, and prior history of radiotherapy, were not significantly different between the two groups, there were significant differences regarding prior history of chemotherapy and the interval from radiation to chemotherapy between the two groups, which can cause several biases in both toxicity profile and clinical outcomes. Third, while the hematological toxicity is not the major concern with vincristine, but rather the peripheral neurotoxicity, our findings shows that hematological toxicities were more frequently occurred in the PCV group than the PC group and that the rates of peripheral neurotoxicity was not different statistically $(0.0 \%$ vs. $11.4 \%, p=0.408)$. This may be due to a smaller dose of lomustine used in the PC group than that of the PCV group $\left(75 \mathrm{mg} / \mathrm{m}^{2} \mathrm{vs} .110\right.$ $\mathrm{mg} / \mathrm{m}^{2}$ ), which is known to be a frequent cause of hematologic toxicity. Due to these several limitations of our study, further prospective and larger studies are needed to validate whether PC chemotherapy could be an alternative to PCV chemotherapy as a secondary salvage option for recurrent glioma patients.

In conclusion, our comparative study involving patients from two institutions showed that toxicities after PC chemotherapy were significantly fewer than those after PCV chemotherapy in recurrent glioma 
patients. The OS and PFS of PC chemotherapy were also noninferior to those of PCV chemotherapy. Further prospective and larger studies are needed to validate whether PC chemotherapy had a better toxicity profile than that of PCV chemotherapy without loss of clinical efficacy in glioma patients.

\section{Declarations}

\section{Conflict of Interest}

Nothing to declare.

\section{Ethics approval}

The Institutional Review Board of Seoul St. Mary's Hospital approved the study design (ethical code: XC21RIDI0089).

\section{Consent to participate}

Due to the retrospective manner of the study, the requirement for informed consent to patriciatewas waived.

\section{Consent for publication}

Due to the retrospective manner of the study, the requirement for informed consent for publication was waived.

\section{Availability of data and material}

Data available on request due to privacy/ethical restrictions.

\section{Funding}

None

\section{Author's contribution}

Stephen Ahn: Writing original draft, data collection \& curation

Young II Kim: Writing original draft, data collection \& curation

Ja Young Shin: Data collection \& curation

Jae-Sung Park: Writing - review \& editing

Changyoung Yoo: Supervision

Youn Soo Lee: Supervision 
Yong-Kil Hong: Supervision

Sin-Soo Jeun: Writing - review \& editing, supervision, conceptualization

Seung Ho Yang: Writing - review \& editing, supervision, conceptualization

\section{References}

1. Ostrom QT, Cioffi G, Gittleman H, Patil N, Waite K, Kruchko C, Barnholtz-Sloan JS (2019) CBTRUS statistical report: primary brain and other central nervous system tumors diagnosed in the United States in 2012-2016. Neuro-oncology 21:v1-v100

2. Lapointe S, Perry A, Butowski NA (2018) Primary brain tumours in adults. The Lancet 392:432-446

3. Molinaro AM, Taylor JW, Wiencke JK, Wrensch MR (2019) Genetic and molecular epidemiology of adult diffuse glioma. Nature Reviews Neurology 15:405-417

4. Weller M, van den Bent M, Preusser M, Le Rhun E, Tonn JC, Minniti G, Bendszus M, Balana C, Chinot $O$, Dirven $L$ (2020) EANO guidelines on the diagnosis and treatment of diffuse gliomas of adulthood. Nature Reviews Clinical Oncology:1-17

5. Stupp R, Hegi ME, Mason WP, Van Den Bent MJ, Taphoorn MJ, Janzer RC, Ludwin SK, Allgeier A, Fisher B, Belanger K (2009) Effects of radiotherapy with concomitant and adjuvant temozolomide versus radiotherapy alone on survival in glioblastoma in a randomised phase III study: 5-year analysis of the EORTC-NCIC trial. The lancet oncology 10:459-466

6. van Linde ME, Brahm CG, de Witt Hamer PC, Reijneveld JC, Bruynzeel AM, Vandertop WP, van de Ven PM, Wagemakers M, van der Weide HL, Enting RH (2017) Treatment outcome of patients with recurrent glioblastoma multiforme: a retrospective multicenter analysis. Journal of neuro-oncology 135:183-192

7. Wen PY, Weller M, Lee EQ, Alexander BM, Barnholtz-Sloan JS, Barthel FP, Batchelor TT, Bindra RS, Chang SM, Chiocca EA (2020) Glioblastoma in adults: a Society for Neuro-Oncology (SNO) and European Society of Neuro-Oncology (EANO) consensus review on current management and future directions. Neuro-oncology 22:1073-1113

8. Alexander BM, Cloughesy TF (2017) Adult glioblastoma. Journal of Clinical Oncology 35:2402-2409

9. Weller M, Le Rhun E (2020) How did lomustine become standard of care in recurrent glioblastoma? Cancer treatment reviews:102029

10. McDuff SG, Dietrich J, Atkins KM, Oh KS, Loeffler JS, Shih HA (2020) Radiation and chemotherapy for high-risk lower grade gliomas: Choosing between temozolomide and PCV. Cancer medicine 9:311

11. Toft A, Urup T, Christensen IJ, Michaelsen SR, Lukram B, Grunnet K, Kosteljanetz M, Larsen VA, Lassen U, Broholm H (2018) Biomarkers in recurrent grade III glioma patients treated with bevacizumab and irinotecan. Cancer investigation 36:165-174 
12. Seystahl K, Hentschel B, Loew S, Gramatzki D, Felsberg J, Herrlinger U, Westphal M, Schackert G, Thon N, Tatagiba M (2020) Bevacizumab versus alkylating chemotherapy in recurrent glioblastoma. Journal of cancer research and clinical oncology 146:659-670

13. Schmidt F, Fischer J, Herrlinger U, Dietz K, Dichgans J, Weller M (2006) PCV chemotherapy for recurrent glioblastoma. Neurology 66:587-589

14. Happold C, Roth P, Wick W, Steinbach JP, Linnebank M, Weller M, Eisele G (2009) ACNU-based chemotherapy for recurrent glioma in the temozolomide era. Journal of neuro-oncology 92:45-48

15. Brada M, Stenning S, Gabe R, Thompson LC, Levy D, Rampling R, Erridge S, Saran F, Gattamaneni R, Hopkins K (2010) Temozolomide versus procarbazine, lomustine, and vincristine in recurrent highgrade glioma. J Clin Oncol 28:4601-4608

16. Parasramka S, Talari G, Rosenfeld M, Guo J, Villano JL (2017) Procarbazine, lomustine and vincristine for recurrent high-grade glioma. Cochrane Database of Systematic Reviews

17. Jutras G, Bélanger K, Letarte N, Adam J-P, Roberge D, Lemieux B, Lemieux-Blanchard É, Masucci L, Ménard C, Bahary J (2018) Procarbazine, lomustine and vincristine toxicity in low-grade gliomas. Current Oncology 25:e33

18. Keogh RJ, Aslam R, Hennessy MA, Coyne Z, Hennessy BT, Breathnach OS, Grogan L, Morris PG (2021) One year of procarbazine lomustine and vincristine is poorly tolerated in low grade glioma: a real world experience in a national neuro-oncology centre. BMC cancer 21:1-8

19. Park SB, Goldstein D, Krishnan AV, Lin CSY, Friedlander ML, Cassidy J, Koltzenburg M, Kiernan MC (2013) Chemotherapy-induced peripheral neurotoxicity: a critical analysis. CA: a cancer journal for clinicians 63:419-437

20. Wang F, Zhou F, Kruh GD, Gallo JM (2010) Influence of blood-brain barrier efflux pumps on the distribution of vincristine in brain and brain tumors. Neuro-oncology 12:1043-1049

21. Kim S-H, Yoo H, Chang JH, Kim C-Y, Chung DS, Kim SH, Park S-H, Lee YS, Yang SH (2018) Procarbazine and CCNU chemotherapy for recurrent glioblastoma with MGMT promoter methylation. Journal of Korean medical science 33

22. Webre C, Shonka N, Smith L, Liu D, De Groot J (2015) PC or PCV, that is the question: primary anaplastic oligodendroglial tumors treated with procarbazine and CCNU with and without vincristine. Anticancer research 35:5467-5472

23. Vesper J, Graf E, Wille C, Tilgner J, Trippel M, Nikkhah G, Ostertag C (2009) Retrospective analysis of treatment outcome in 315 patients with oligodendroglial brain tumors. BMC neurology 9:1-9

24. Yang S-H, Hong Y-K, Yoon S-C, Kim B-S, Lee Y-S, Lee T-K, Lee K-S, Jeun S-S, Kim M-C, Park C-K (2007) Radiotherapy plus concurrent and adjuvant procarbazine, lomustine, and vincristine chemotherapy for patients with malignant glioma. Oncology reports 17:1359-1364

25. Cha J-Y, Park J-S, Hong Y-K, Jeun S-S, Ahn S (2021) Impact of Body Mass Index on Survival Outcome in Patients with Newly Diagnosed Glioblastoma: A Retrospective Single-Center Study. Integrative Cancer Therapies 20:1534735421991233

Page 13/16 
26. Trestini I, Carbognin L, Bonaiuto C, Tortora G, Bria E (2018) The obesity paradox in cancer: clinical insights and perspectives. Eating and Weight Disorders-Studies on Anorexia, Bulimia and Obesity 23:185-193

27. Cai Y, Jiang Y-G, Wang M, Jiang Z-H, Tan Z-G (2020) A comparative study of the effectiveness and safety of combined procarbazine, lomustine, and vincristine as a therapeutic method for recurrent high-grade glioma: A protocol for systematic review and meta-analysis. Medicine 99

28. Wick W, Winkler F (2018) Regimen of procarbazine, lomustine, and vincristine versus temozolomide for gliomas. Cancer 124:2674-2676

29. TABOURET E, REYES-BOTERO G, DEHAIS C, DAROS M, BARRIE M, MATTA M, PETRIRENA G, AUTRAN D, DURAN A, BEQUET C, DELATTRE J-Y, CHINOT O (2015) Relationships Between Dose Intensity, Toxicity, and Outcome in Patients with Oligodendroglial Tumors Treated with the PCV Regimen. Anticancer Research 35:2901-2908

\section{Figures}

75 patients with recurrent adult glioma who received PCV or PC chemotherapy in our institutions between January 2009 and December 2020 was examined.

Among 59 patients who met our eligibility criteria, 15 patients received $\mathrm{PC}$ chemotherapy ( $\mathrm{PC}$ group) and 44 patients received PCV chemotherapy (PCV group).

- Exclude patients who received PCV or PC chemotherapy as adjuvant therapy after surgery. $(\mathrm{n}=15)$

- Exclude patients who were not followed up after one cycle of chemotherapy. $(n=1)$

Evaluate the toxicity profile between PC and PCV groups. $(\mathrm{n}=59)$

- Analysis OS $(n=59)$ and PFS $(n=52)$ of PC and PCV group.

\section{Figure 1}

Flow of the study design. 
(a)

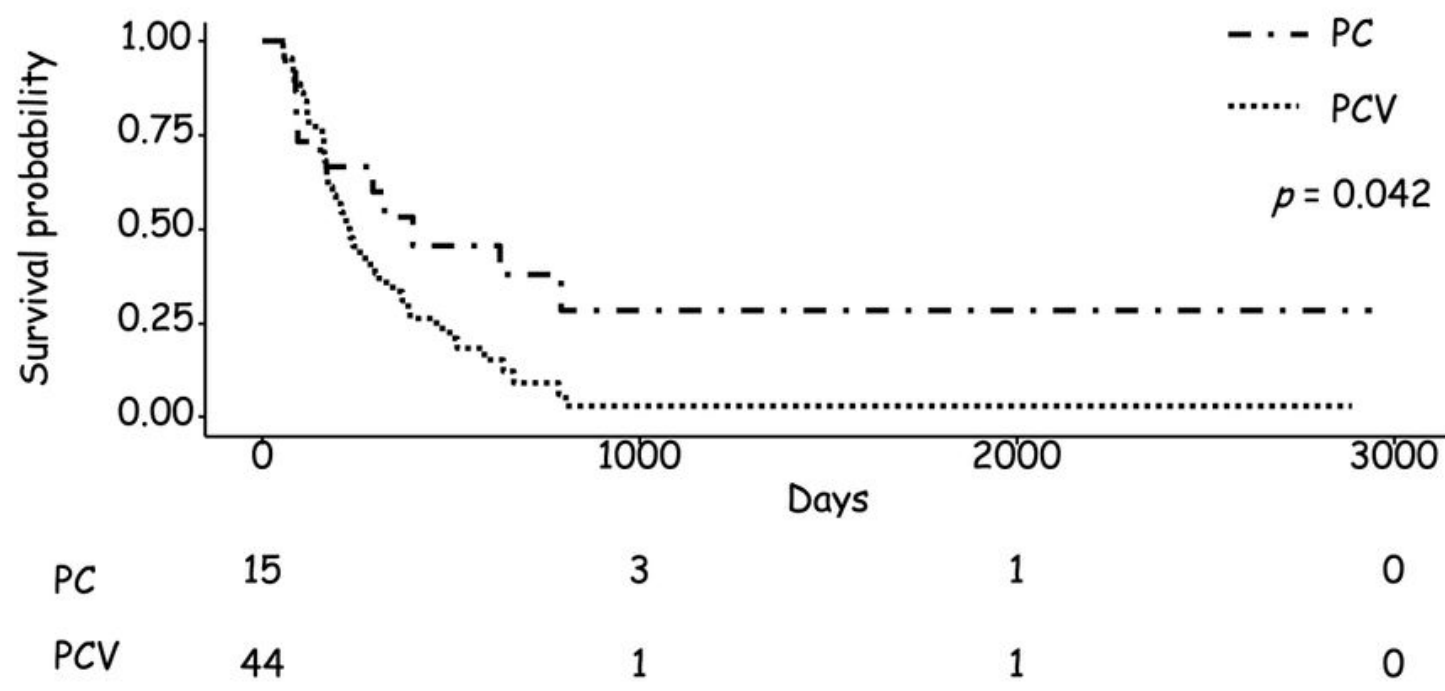

Number at risk

(b)

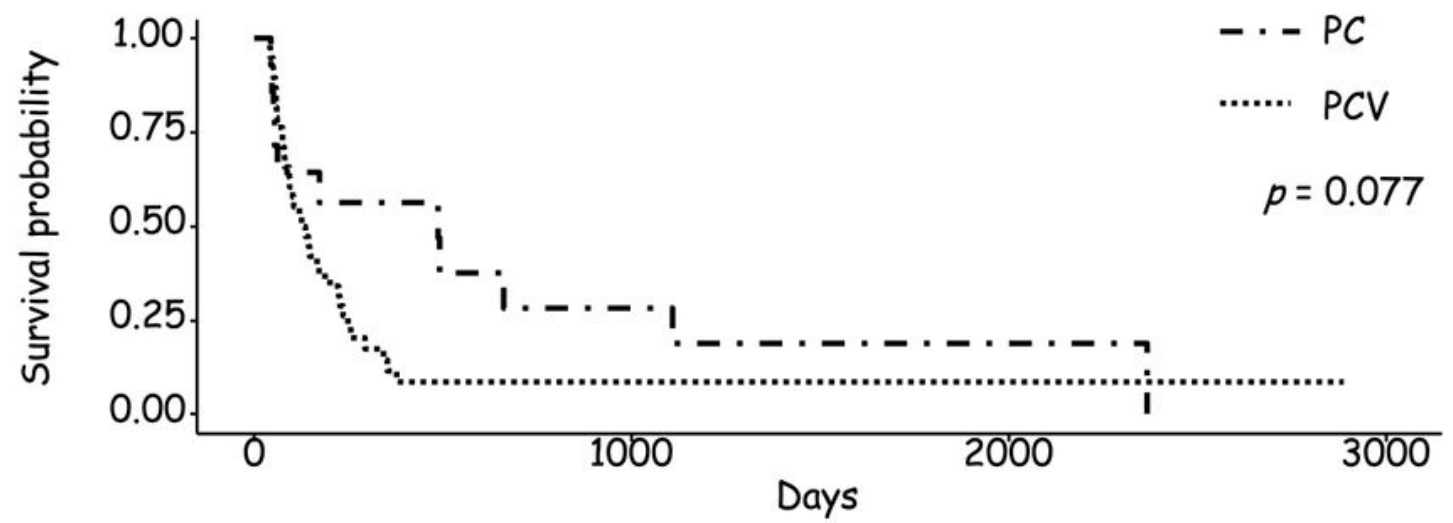

$\begin{array}{lllll}\text { PC } & 14 & 3 & 1 & 0 \\ \text { PCV } & 38 & 1 & 1 & 0\end{array}$

Number at risk

Figure 2

(a) OS and (b) PFS of the PC and PCV groups.

\section{Supplementary Files}

This is a list of supplementary files associated with this preprint. Click to download. 
- sFigure1.jpg

- sFigure2.jpg

Page 16/16 